vol. $19-n^{\circ} 2$ | 2003

Les initiatives de l'étranger et les nouveaux

cosmopolitismes

\title{
Des migrants et des squats : précarités et résistances aux marges de la ville
}

Florence Bouillon

\section{(2) OpenEdition}

Journals

Édition électronique

URL : https://journals.openedition.org/remi/440

DOI : $10.4000 /$ remi.440

ISSN : $1777-5418$

Éditeur

Université de Poitiers

Édition imprimée

Date de publication : 1 juillet 2003

Pagination : 23-46

ISBN : 2-911627-34-2

ISSN : 0765-0752

Référence électronique

Florence Bouillon, « Des migrants et des squats : précarités et résistances aux marges de la ville », Revue européenne des migrations internationales [En ligne], vol. 19 - n² | 2003, mis en ligne le 19 avril 2007, consulté le 14 avril 2022. URL : http://journals.openedition.org/remi/440 ; DOI : https://doi.org/ $10.4000 /$ remi.440

Ce document a été généré automatiquement le 14 avril 2022.

(c) Université de Poitiers 


\title{
Des migrants et des squats : précarités et résistances aux marges de la ville
}

\author{
Florence Bouillon
}

1 La question du logement des migrants ${ }^{1}$ ne fait pas partie des interrogations qui se posent aujourd'hui dans le champ politique français. Si des dispositifs spécifiques d'accès au logement ont existé par le passé ${ }^{2}$ ils ont peu à peu été abandonnés au profit de dispositions de droit commun, au nom d'un «modèle d'intégration à la française » qui ne reconnaît aucune identité collective et refuse toute discrimination, serait-elle positive.

2 Pourtant, les inégalités face au logement existent bien. Les migrants en provenance des pays pauvres, dont on a longtemps pensé qu'ils suivraient les trajectoires résidentielles des classes populaires françaises passées des HLM aux maisons individuelles dans les années 1970-1980, se retrouvent en réalité cantonnés aux étages les plus bas du marché du logement : le privé dégradé, et le locatif social ${ }^{3}$.

On connaît assez précisément les conditions de vie, les problèmes liés à la ségrégation, les formes de violence et de sociabilités des banlieues et cités que les migrants peuplent à présent, et que nombre de chercheurs ont pris comme terrains d'enquête et objets d'étude. On sait moins ce qu'il advient de ceux qui n'accèdent pas du tout au logement de droit commun. C'est à eux, et à la manière dont ils aménagent leur précarité, que l'on voudrait par conséquent réfléchir.

4 Cet article propose une description des modes d'habitat d'individus inscrits dans des circulations transfrontalières, et dont les trajectoires résidentielles sont marquées par l'exclusion du logement standard. Les diverses situations rencontrées dans ce cadre peuvent être classées en trois types: l'exploitation, la précarité et l'illégalité. La construction de cette typologie, qui n'a rien d'exclusif ni de définitif, s'indexe essentiellement sur deux paramètres, qui sont le degré d'autonomie ou de dépendance des individus, et le coût psychologique et financier de leur mode d'hébergement. 
5 Après avoir précisé ce que recouvrent ces termes, on travaillera plus précisément la question de l'illégalité, c'est-à-dire celle du squat, entendu comme occupation sans droit ni titre d'un appartement ou d'un bâtiment vacant. Cette analyse prend appui sur des observations effectuées au cours des cinq dernières années au sein d'une vingtaine de squats à Marseille, accompagnées de temps de résidence dans certains, et présentées ici sous forme de récits et d'extraits d'entretiens. À travers eux, nous rencontrerons d'autres figures sociales que les migrants paupérisés, en particulier celle de jeunes européens vivant sur un mode collectif au sein de ce que nous appelons les squats d'activités, destinés à l'art, à la politique et au voyage.

6 Ces descriptions nous indiquent donc la place du squat dans une histoire migratoire, le moment et les circonstances du passage à l'illégalité, mais aussi la très grande hétérogénéité des squats et de leurs habitants. Si le squat apparait alors comme prisme d'analyse des formes de mobilité précaire contemporaines, et de la manière dont elles s'inscrivent dans les interstices de nos sociétés, on perçoit qu'il n'est pas seulement révélateur des mécanismes de production de la misère. Son caractère éminemment autonome, dans le sens d'une indépendance de fait à l'égard des structures institutionnelles, révèle des compétences et des ressources inattendues ${ }^{4}$, et permet des formes de mixité sociale tangibles et ignorées. Espace interlope, il provoque des rencontres entre divers mondes sociaux que les instances décisionnelles appellent de leurs vœux, et ignorent dans le même temps lorsqu'elles se produisent en dehors des circuits programmés de la sociabilité et de la mixité. Au final, le squat peut être envisagé comme un lieu de résistance ${ }^{5}$, dont on déclinera ici les diverses modalités.

Exploitation

7 Il n'est qu'à travailler de visu à Marseille sur l'exclusion du logement pour constater que les migrants en sont les principales victimes ${ }^{6}$. Beaucoup sont des primo-arrivants issus des migrations les plus récentes, en provenance des pays d'Europe de l'Est et d'Afrique forestière. D'autres arrivent aujourd'hui à la suite d'une longue histoire migratoire, du Maghreb (Algérie en particulier), d'Afrique sub-saharienne et des Comores. L'instauration successive de nouvelles catégories de logement pour les plus démunis au cours des dix dernières années n'a donc pas permis aux plus pauvres et aux plus stigmatisés d'y accéder'.

8 Le schème de l'exploitation recouvre des situations de très grande fragilité et d'insécurité. La dépendance à l'égard d'une tierce personne, logeur ou parent abusif, est au cœur de ce type d'habitat. Il concerne en priorité les migrants en situation irrégulière, ainsi que des familles possédant des cartes de séjour mais exclues du logement de droit commun, en raison d'une impossibilité à trouver quelqu'un pour se porter caution, et du «tri urbain ${ }^{9}$ effectué par les bailleurs sociaux et les propriétaires privés.

9 Deux types d'habitations correspondent à ce modèle : des appartements ou des hôtels meublés loués par des "marchands de sommeil»; l'hébergement chez des proches lorsqu'il est essentiellement motivé par l'intérêt.

10 Il existe en effet une demande massive de logements inassouvie, un marché potentiel de la pauvreté que les «marchands de sommeil » ont saisi. Cette pratique est courante à Marseille, notamment dans les quartiers centraux populaires, et dans certaines cités périphériques. Le fonctionnement en est simple: des propriétaires font l'acquisition d'appartements ou d'hôtels qu'ils laissent se dégrader, et les louent à des individus ne pouvant accéder au logement standard, dans l'immense majorité des cas une 
population d'origine étrangère démunie. Les appartements, souvent petits et extrêmement vétustes, sont en partie financés par le biais des allocations-logements, payées directement au propriétaire, selon le système du tiers-payant. Les Caisses d'Allocations Familiales en effet attribuent les allocations uniquement en fonction des ressources et de la composition des ménages, sans contrôler l'état des logements ${ }^{10}$.

11 À Marseille, D. Sanchez possède 112 appartements vétustes dans le Panier, quartier ancien proche du Vieux-Port, qui lui rapportent chaque mois plus de 30000 euros $^{11}$. On sait que ces appartements sont frappés d'arrêtés d'insalubrité, certains depuis 1991. Mais comme pour les hôtels insalubres du centre-ville dans lesquels habitent, depuis parfois des décennies, de vieux Maghrébins à la retraite ${ }^{12}$, les situations perdurent, les pouvoirs publics étant réticents à devoir gérer le relogement de centaines d'individus dont ils savent qu'ils seront refusés par les logeurs sociaux. Les locataires quant à eux se taisent, redoutant de se retrouver à la rue.

12 L'hébergement chez des proches, famille ou amis, peut quant à lui se dérouler de manière très diverse, en fonction des liens unissant les individus, des motivations des hôtes et des ressources dont disposent les hébergés. Ainsi G. Engbersen (1999) fait-il la distinction entre les migrants qui disposent d'un capital social, d'une forte insertion dans un groupe susceptible de faire pression sur les proches ou encore d'une famille agissant essentiellement par altruisme, et ceux pour lesquels l'aide apportée ne repose que sur l'intérêt et entraîne un endettement moral et financier conséquent.

13 En règle générale l'hébergement chez des proches, vécu comme provisoire il y a 25 ans lorsque les chances de trouver un emploi légal et un conjoint étaient fortes, est aujourd'hui fréquemment source de tensions: la durée d'hébergement s'allonge, sans perspective de changement, et la fragilité des individus sollicités comme l'affaiblissement des liens de solidarité conduisent à des situations de dépendance, de paternalisme, d'utilisation de la force de travail de l'individu considéré comme corvéable à merci, de prostitution parfois. Le fait d'être redevable est constamment rappelé à ceux qui n'ont dès lors plus guère de latitude pour se forger une identité autre que celle, imposée, de dominé. Le logement lié à l'exploitation a donc un coût psychologique lourd, et il n'est jamais gratuit, alors même qu'il concerne les migrants les plus dépourvus économiquement.

Précarité

14 La précarité désigne l'ensemble des situations au sein desquelles le sentiment de dépendance est moins violemment ressenti que dans l'exploitation, mais où l'instabilité, la dimension provisoire et l'insatisfaction restent prédominantes.

Quatre cas de figure sont alors possibles : l'hébergement chez des proches ou dans un hôtel, lorsque les conditions d'accueil et de vie y sont moins aliénantes que précédemment, le centre d'hébergement et le foyer de travailleur.

16 Les deux premiers cas concernent des individus socialement proches de ceux qui sont victimes de l'exploitation, mais pour diverses raisons leur assujettissement est moindre. Une association finance par exemple des hôtels mieux équipés que ceux qui sont loués par les marchands de sommeil, tout en assurant un minimum de suivi social. Les migrants disposent d'un travail, d'un peu d'argent, de davantage de relations, d'un accès aux droits sociaux..., qui atténuent leur position de subordination vis-à-vis de leur logeur ou de leur famille, qui exige également moins de contrepartie à leur présence. Si la situation est toujours vécue - ou espérée - comme transitoire, elle est 
moins difficile à vivre moralement (et financièrement dans le cas des marchands de sommeil).

Les foyers d'urgence ou de réinsertion et les foyers de travailleurs du type Sonacotra ${ }^{13}$ constituent les deux autres formes de logement précaire. L'hébergement d'urgence est limité dans le temps, contraignant (respect des horaires, des normes comportementales, cohabitation avec des individus très désaffiliés...) et n'est pas apte à recevoir des familles; il incarne probablement le plus fragile et le plus disqualifiant des habitats de cette catégorie. Quant aux CHRS $^{14}$ et appartements-relais gérés par des associations, ils sont réservés aux détenteurs de titres de séjour: les personnes en situation irrégulière n'y ont, par conséquent, pas accès.

Les foyers de travailleurs hébergent eux aussi des étrangers détenteurs de papiers. Ils accueillaient initialement des ouvriers maghrébins appelés par la France, aujourd'hui vieillissants, et concurrencés par d'autres catégories de personnes auxquelles les foyers ouvrent désormais leurs portes : étudiants, chômeurs, RMIstes ${ }^{15}$, femmes célibataires, jeunes travaillant en intérim, à temps partiel, en contrat à durée déterminée. Bien qu'ils se soient nettement améliorés en termes de confort et de prestation offertes avec la sédentarisation de leurs clients, les foyers de travailleurs sont toujours assimilables à du logement précaire, habitat collectif sans possibilité de vie familiale, qui, lorsqu'elle existe, se résume souvent aux quelques semaines passées chaque année dans le pays ou la région d'origine.

19 Au sein du logement précaire, deux types d'hébergements sont donc gratuits : celui assuré par les proches, qui n'exigeront pas de contrepartie immédiate ou différée, et celui en foyer d'urgence ou de réinsertion ${ }^{16}$. Les deux autres, l'hôtel et le foyer pour travailleur migrant nécessitent la perception d'un minimum de revenus, qu'ils soient issus du travail ou des aides sociales.

Illégalité

20 L'illégalité enfin désigne l'occupation sans droit ni titre d'un logement, c'est-à-dire le squat ${ }^{17}$. Le terme squatter (1835) vient de l'anglais des États-Unis, et désigne à l'origine les pionniers qui s'installaient sur les terres inexploitées de l'Ouest américain, sans payer de redevance. Aujourd'hui, il qualifie communément une personne sans logement qui occupe illégalement et gratuitement un bâtiment vacant ${ }^{18}$.

21 Le squat apparaît d'abord comme le dernier maillon de la chaîne de la galère, celui auquel on ne se résout qu'en dernière instance et en désespoir de cause. Et de fait, l'occupation illégale est source d'un sentiment justifié d'insécurité et d'instabilité, mais aussi d'un inconfort moral pour ceux qui, nombreux, aspirent à respecter les règles $\mathrm{du}$ jeu en vigueur en France, et redoutent le passage à l'illégalité. Le squat est alors vécu comme une menace, à laquelle on ne se résoudra, difficilement, que s'il n'y a pas d'alternative. L'analyse des trajectoires résidentielles des squatters et du traitement juridique des squats nous révèle ainsi des situations caractérisées par un choix très restreint, une dépendance éventuelle vis-à-vis d'autres squatters, et un coût, psychologique ou même financier, élevé.

Pourtant, force est de constater que le vécu des squatters est foncièrement hétérogène, et que le squat est également synonyme d'autonomisation, et de création. On verra dans un deuxième temps que les squatters sont aussi en mesure de nous indiquer la manière dont des individus habitent la ville en dehors des chemins tracés par les décideurs, 
aménageurs et urbanistes, et comment se tissent des liens, divers et chaotiques, au sein des mondes de la déterritorialisation.

Le squat en bout de course nous reviendrons. Karima est assistance sociale dans le CHRS qui les héberge, et a fait connaissance quelques semaines plus tôt d'un occupant de l'Huilerie. Lorsque plus 
aucune solution de logement ne peut être trouvée, Karima décide de faire la médiation entre la famille et le squat.

$\mathrm{Au}$ cours de leur première visite à l'Huilerie, les Ramdani ne parlent guère ; ils semblent mal à l'aise, intimidés par les squatters, et psychologiquement fatigués. Karima explique que la famille est « en bout de course »:

«Ils ont tout essayé, essayé toutes les structures, on a tout fait. Le squat est hyper mal vu par les travailleurs sociaux en général. Et aux Ramdani, ça leur fait très peur. Mais là, on n'a plus du tout le choix. On risque notre place si ce qu'on fait est ébruité (...) Ce sont des gens très intègres, rigoureux, qui n'ont pas du tout envie de se retrouver dans l'illégalité ! Ils ont très peur que cela leur cause des problèmes supplémentaires. Mais c'est la rue ou le squat. Alors il vaut mieux le squat. Surtout que dans la rue, les enfants risquent d'être placé par la DDASS, ce qui est la grande peur des parents. »

Le squat politique de l'Huilerie n'a pas pour vocation d'accueillir des personnes en difficulté, mais des militants ${ }^{22}$. Il n'est donc pas question d'héberger la famille sur place. Après bien des discussions, un petit groupe décide finalement d'ouvrir pour elle un appartement que l'on sait vacant, à proximité du quartier Belsunce.

31 Les réticences de la famille, les hésitations des avocats interrogés sur l'éventualité d'une aggravation de leur situation administrative du fait de l'occupation illégale d'un logement nous incitent tout de même à poursuivre nos recherches. Après de multiples coups de téléphone, et des refus tout aussi nombreux, nous finissons par trouver une association caritative catholique qui, non subventionnée, héberge des individus «sans papiers ». Les Ramdani iront donc s'installer là-bas, évitant pour finir le squat qu'ils redoutaient tant.

$32 \quad A u$ bord du squat, les Ramdani appréhendaient cette situation plus que d'autres, parce que accompagnés de nombreux enfants, inquiets des répercussions que le squat pourrait avoir sur leur situation administrative, et très attachés à la moralité qu'ils associent à la légalité. Mais leur histoire ressemble à celle d'innombrables familles et individus qui squattent en désespoir de cause.

33 Les conditions du squat peuvent alors varier considérablement. Plus encore peut-être que les autres types de logement évoqués, les squats sont d'une très grande hétérogénéité ; on a pressenti, avec le récit exposé ci-dessus, qu'ils hébergeaient des mondes sociaux et culturels fort éloignés, et offraient des conditions de vie, de confort et de sécurité extrêmement divers. Les squats les plus éphémères et insalubres au sein de cette nébuleuse sont probablement ceux des mineurs clandestins, qui à Marseille viennent pour la plupart du Maghreb ${ }^{23}$. Arrivés en France après des conditions de voyage souvent extrêmes, ces jeunes migrants pensent y trouver du travail, de l'argent, une autre vie que celle à laquelle le bled et le dénuement ambiant les destinaient. C'est bien sûr la déconvenue; le travail escompté fait place à des pratiques délinquantes, essentiellement le vol, à la tire ou à la roulotte.

Les migrants de ce type incarnent l'image paroxystique d'individus n'ayant aucune chance d'accéder au logement standard: ils sont mineurs, issus de familles pauvres ${ }^{24}$, isolés, en situation irrégulière, insolvables, assimilés à des "sauvageons " et très fortement stigmatisés. Certains sont hébergés temporairement chez des proches, d'autres en hôtels meublés, une partie au sein de foyers d'éducation spécialisée. Le squat constitue une autre possibilité de logement, mais il est fréquemment trouvé et ouvert par des hommes maghrébins plus anciens à Marseille, et qui exigeront des 
mineurs une rémunération afin de pouvoir dormir dans le lieu squatté: le squat est alors payant.

Les appartements occupés par les mineurs clandestins sont assimilables à des taudis, dans lesquels ont été installés des matelas dans le meilleur des cas, et où des sacs et bouteilles en plastique font office de sanitaires. Ici les portes ne sont pas fermées, ce qui engendre pour les habitants du lieu un climat permanent d'insécurité; l'eau et l'électricité ne sont pas branchées, par manque de compétences, peur de représailles judiciaires ou simple indifférence des "propriétaires de squats». Les expulsions interviennent au bout de quelques semaines, voire quelques jours, et l'assurance de devoir bientôt « déguerpir " 25 interdit tout investissement durable dans le lieu.

Ce système de squat payant concerne tous les migrants les plus exposés aux violences de la misère, et l'on a recueilli sur le terrain des récits de femmes sénégalaises, comoriennes et maghrébines contraintes de payer plusieurs milliers de francs l'ouverture d'un squat. L'accès à celui-ci, mais aussi les conditions de résidence dans ce lieu ne sont donc pas les mêmes pour tous.

Les inégalités de l'illégalité

37 Au-delà de cette dimension payante du squat, et de l'insalubrité, le plus grand facteur de fragilité des squatters réside dans leur méconnaissance des droits et des quelques protections juridiques dont ils disposent, et parallèlement dans la manière dont la police et la justice appliquent ou non ces règles de droit.

L'occupation sans droit ni titre n'est pas passible de condamnation pénale ; tout au plus le squatter peut-il être assigné à payer une amende s'il y a eu effraction, ou dégradation manifeste des lieux. Le risque majeur qu'il encourt est donc celui d'une expulsion rapide $^{26}$. Or selon l'article 61 de la loi du 9 juillet 1991, "sauf disposition spéciale, l'expulsion ou l'évacuation d'un immeuble ne peut être poursuivie qu'en vertu d'une décision de justice ou d'un procès-verbal de conciliation exécutoire, et après commandement d'avoir à libérer les locaux. » Cette procédure assure théoriquement quelques semaines de répit aux squatters, et plusieurs mois peuvent s'écouler avant que le commandement à quitter les lieux ne soit délivré.

Dans les faits, les propriétaires se passent autant que faire se peut de procédure juridique. Certains, à la limite de la légalité, utilisent l' " ordonnance sur requête », qui permet de demander le concours de la force publique sans que les squatters ne soient avertis de la procédure en cours ${ }^{27}$. D'autres cherchent à les intimider, en proférant des menaces de dénonciation auprès de la police ou de la justice. Peu au fait de leurs droits, ou inquiets des répercussions de cette dénonciation sur le cours de leurs démarches administratives, les plus fragiles partiront. Les propriétaires usent aussi de représailles physiques, par le biais de "gros bras » qu'ils mobilisent pour les mettre dehors. Cette pratique, parfaitement illégale, s'apparente à une « violation de domicile $»^{28}$, beaucoup plus grave en termes de sanction juridique que ne l'est le squat. Mais en dehors d'un cas avéré de coups et blessures portés à des jeunes gens qui ont dû être hospitalisés et ont porté plainte, les squatters rencontrés s'étant fait chassés de la sorte n'ont jamais saisi la justice.

40 On sait également d'après l'enquête de terrain que la police expulse les occupants de squats sans respecter les obligations de procédure préalable. Aux dires d'un commandant de la police nationale, les individus isolés et les « délinquants » sont mis 
dehors manu militari, sans appel à huissier, sans jugement, sans qu'aucune procédure légale ne soit suivie.

«Les squatters, ce sont des personnes marginales (...) Nous les appréhendons généralement à l'extérieur, on trouve un motif bidon pour intervenir, et on contrôle la personne. Si c'est un squat de plusieurs personnes, la ville coopère, l'eau et l'électricité sont coupées. Si le propriétaire ne dit rien, qu'il n'y a pas de plainte, on trouve des artifices plus ou moins légaux pour intervenir.

- Et les expulsions, comment se déroulent-elles?

- Ah, mais vous m'avez demandé de parler des squats, pas des expulsions! Ça n'a rien à voir ! Les expulsions, c'est pour les familles, qui ont signé un bail, mais ont un problème d'argent et ne peuvent plus payer (...) Rares sont les squats familiaux. Pour une famille, le côté humain est pris en compte, on recherche un relogement. Mais il n'y a pas de mesure de relogement pour les squatters marginaux. On invente un peu la situation, on interpelle la personne sur la voie publique, et puis on rentre... Lorsqu'ils sont en interpellation ou dehors, une benne de la ville vient et débarrasse tout. Puis on mure. »

41 Les pratiques diffèrent donc selon le degré de visibilité et de légitimité des squatters. Les familles sont mieux tolérées que les hommes seuls ou les groupes, les Français que les migrants, les vieux que les jeunes.

Les propositions de relogement suite à une expulsion sont quant à elles faites à ceux qui sont en mesure de faire peser des menaces de médiatisation face à une institution qui n'en veut pas, ou à ceux qui sont en capacité de se présenter de manière conforme aux normes en vigueur : en situation régulière, occidental de préférence, sans dépendance toxicologique ou autre comportement déviant avéré. Les relogements sont plus courants au sein des offices HLM que dans le parc privé. Mais elles sont loin d'être systématiques et demeurent globalement très minoritaires. D'autre part, il semblerait que les squatters expulsés du parc social locatif aient les plus grandes difficultés à le réinvestir de manière légale. Deux arguments principaux sont avancés par les bailleurs sociaux pour justifier leur refus de les reloger: celui du «mauvais exemple», leur relogement étant susceptible d'être interprété comme un encouragement à squatter par d'autres; et celui de l'» injustice » que constituerait le fait de privilégier des occupants sans droit ni titre sur les ménages en attente d'un appartement. Une militante de la $\mathrm{CSF}^{29}$, habitante d'un grand ensemble et très engagée sur le problème du mal-logement, confirmait lors d'un entretien que la grande majorité des squatters des cités font des demandes auprès des $\mathrm{HLM}^{30}$ mais n'obtiennent pas de réponse, ce qu'elle résumait par cette formule lapidaire : « les bailleurs ne veulent pas passer pour des cons ».

La justice, lorsqu'elle est sollicitée, effectue à son tour une distinction entre les «bons » squatters, "vrais " pauvres inoffensifs, et les «mauvais », associés aux délinquants de tout ordre.

Un juge a fait grand bruit à Marseille au début de l'année 2001 en accordant un délai d'un an avant expulsion à des squatters, en se basant sur l'argument d'une carence massive en matière de logement social dans la ville. C'est là d'une part accorder un délai bien supérieur à ce que les magistrats ont coutume d'octroyer ${ }^{31}$, et d'autre part reconnaître que la collectivité, et en particulier les pouvoirs publics en charge de la politique du logement, ont une part de responsabilité dans cette situation.

Arrêtons-nous sur les squatters dont il est ici question. À l'initiative de plusieurs associations dont Droit Au Logement (DAL), 46 familles d'origine comorienne (200 personnes dont 143 enfants), sans domiciles ou mal logées, ont quitté au mois de février 2001 les appartements insalubres et les taudis qu'elles habitaient pour occuper le siège 
social de HMP dans la cité Frais Vallon (15ème). Une partie d'entre elles avaient été expulsées de logements qu'elles occupaient (légalement) dans cette cité en mars 1998, et le maire de la ville avait promis des relogements rapides qui ne viennent pas.

Expulsées à nouveau lors de leur occupation d'HMP, les familles ont installé un campement de fortune devant ces mêmes locaux. Demande est faite à la Préfecture de les reloger en urgence. La Préfecture attribue 19 logements, et propose une inscription au Plan départemental pour le logement pour les autres. Devant le refus réitéré d'accorder davantage, les familles investissent un immense bâtiment situé rue Lafon, à proximité de cette même Préfecture. Le bâtiment est un ancien centre hospitalier inoccupé depuis 1994, qui appartient à l'Assistance Publique - Hôpitaux de Marseille. Elle demande l'expulsion immédiate.

Au cours de l'audience en référé du 16 mars 2001, l'avocat de l'Assistance Publique avance que celle-ci n'a pas pour mission de traiter les problèmes de logement, et que l'occupation de ses locaux pose des problèmes de sécurité et d'hygiène. L'avocat des squatters conteste ce dernier point, invitant le magistrat à venir établir lui-même sur place l'état des lieux. Il évoque ensuite l'opacité et les multiples irrégularités constatées lors des attributions de logements $\mathrm{HLM}^{32}$, et revient sur les discriminations ethniques dont les familles seraient victimes. Il souligne que bien que démunies, presque toutes sont solvables, mais qu'elles n'ont pas de relation pouvant se porter caution pour la signature d'un bail ${ }^{33}$.

Le juge ordonne finalement l'expulsion des squatters, mais leur accorde donc un an de délai avant expulsion, arguant d'une inadéquation criante entre l'offre et la demande de logements à Marseille. Il ajoute que ce délai pourra être prorogé en fonction de la situation des habitants à la date d'expulsion. Un an après, la quasi-totalité des familles est relogée, mais quelques-unes ont dû, selon le DAL, repartir dans leurs taudis.

L'encadrement associatif, articulé au travail de l'avocat, permet d'instaurer un rapport de force plus favorable aux squatters que dans le cas d'une occupation isolée. Il assure un gain de légitimité auprès du juge, et d'efficacité en matière de relogement.

Cet encadrement a parallèlement un impact sur la manière dont est perçu et vécu le squat par ses occupants. Les associations, rodées à ce genre d'action, fournissent une aide logistique pour l'ouverture du lieu, puis pour l'installation (apport de mobilier, d'ustensiles, branchement de l'eau et de l'électricité), afin que les conditions de vie quotidiennes y soient décentes. La présence sur place des militants rassure également les squatters, et réduit l'anxiété qui accompagne le fait d'occuper sans droit ni titre. Enfin, les militants maitrisent et transmettent le discours de justification du passage à l'illégalité, qui présente le squat comme action légitime d'émancipation face au mallogement, et comme outil de revendication politique.

Squats d'activités et mixités

51 À l'image des occupations orchestrées par le DAL, d'autres squats sont en quête de visibilité et de relais extérieurs. Mais ceux que nous allons évoquer maintenant ont pour caractéristique d'être des squats d'activités, conçus par leurs habitants comme des espaces de création, de réunion et d'expérimentation dans les champs politiques, sociaux, culturels et artistiques. Lieux de rencontres et d'échanges, ils accueillent un public qui vient participer à des ateliers, concerts, repas, organisés par les squatters. Ce qui est mis en avant ici n'est donc plus la dimension du logement, mais celle de la pratique alternative, et de l'occupation collective ${ }^{34}$. 
52 Les individus impliqués dans la nébuleuse des squats d'activités font généralement la distinction entre les squats politiques et les squats artistiques. Outre la fonction première du lieu, la différenciation s'opère autour de la question des relations entretenues avec les sphères institutionnelles. Schématiquement, car des nuances et exceptions existent, les militants refusent catégoriquement de dialoguer avec les pouvoirs publics, et d'envisager un passage à la légalité. L'objectif est de créer des espaces autonomes et autogérés échappant à tout contrôle institutionnel, et tout compromis équivaut à une compromission. Les habitants des squats artistiques à l'inverse aspirent à trouver un accord avec les propriétaires des lieux occupés, pour pouvoir y demeurer et continuer à travailler dans les ateliers qu'ils ont installés. Ils sollicitent les instances décisionnelles pour qu'elles interviennent en leur faveur, et plus largement pour qu'elles leur accordent une forme de reconnaissance artistique et culturelle ${ }^{35}$.

53 Le squat marseillais du SLAAF, jeu de mot autour du label SDF qui signifie Sans Local d'Activité Artistique Fixe, appartient plutôt à cette seconde catégorie. Ses occupants initiaux sont des artistes de différents domaines, qui se sont constitués en association, et ont tenté de négocier avec la municipalité propriétaire du bâtiment leur maintien dans les lieux ${ }^{36}$. Le SLAAF naît surtout d'une envie commune d'ouvrir un lieu dans le quartier du Panier où les artistes peuvent rencontrer les habitants, discuter, imaginer avec eux des projets culturels. L'occupation d'une ancienne entreprise de menuiserie qui s'ensuivit relève davantage de l'opportunité que d'une volonté délibérée d'occuper sans droit ni titre : le lieu est vaste, vide depuis dix ans, et l'un des futurs squatters a obtenu par l'intermédiaire d'un voisin la clé de la porte d'entrée.

En novembre 2000 une dizaine de personnes, majoritairement des hommes, s'installent donc dans une friche industrielle du Panier. Le logement se fait dans des conditions précaires: l'eau est branchée, mais il n'y a pas de douche, la cuisine est réduite à quelques plaques chauffantes. Certains n'ont pas d'alternative et s'installent immédiatement dans le squat, alors que d'autres conservent un appartement.

L'hétérogénéité socioculturelle de ce groupe annonce déjà la diversité qui caractérisera l'existence du lieu. Si tous sont artistes et ont autour de trente ans, à l'exception d'un journaliste algérien en exil plus âgé, les occupants ne sont pas originaires des mêmes villes ni pays, et ont des trajectoires scolaires et sociales très différentes : certains font des études supérieures et d'autres sont RMIstes, les uns sont issus de l'immigration, les autres non. Les profils sociologiques des individus se laissent difficilement saisir, à l'image de ce jeune homme, diplômé des Beaux-arts, qui dormait dans la rue avant d'intégrer le squat.

Le SLAAF se veut donc d'abord et avant tout un lieu de création en relation avec les habitants du quartier. Les liens se tissent progressivement avec les associations, qui offrent des coups de main, comme le prêt gracieux d'une photocopieuse pour imprimer les invitations aux soirées du SLAAF, le centre social, qui emploiera le journaliste sur un Contrat Emploi Solidarité, les commerçants, qui acceptent de retarder leur fermeture lorsque le squat organise des fêtes nocturnes. Le SLAAF peut se prévaloir d'être devenu au fil des mois un haut lieu d'animation du Panier, organisant des activités destinées aux enfants, des concerts, des repas de quartier, des fêtes pour le Ramadan et l'Aïd auxquelles participent effectivement les habitants.

57 Mais cette ouverture a un coût : celui de la (relative) tranquillité dont bénéficie un lieu plus fermé. La cohabitation des mondes sociaux évoquée ne se fait pas sans heurt. Les 
voisins qui occupent les locaux au-dessus du squat sont des artistes officiels qui louent leurs ateliers à la mairie : eux n'apprécient pas les occupants, et le leur font savoir. Cette cohabitation est particulièrement difficile avec les "jeunes» du Panier, expression vernaculaire qui désigne les adolescents d'origine maghrébine qui évoluent dans le quartier. Ces jeunes hommes, qui ne se mélangent pas aux «filles", sont majoritairement en rupture d'études, et organisent divers petits trafics qui vont du deal de cannabis au vol de Vespas. Un large groupe de jeunes occupe habituellement la place située devant le squat, où ils disposent d'un café dans lequel ils passent plusieurs heures de la journée. La distance sociale entre eux et les squatters est grande, et malgré leurs tentatives, ces derniers ne réussiront jamais à les intéresser à leurs activités. Mais leurs rapports vont cependant très nettement évoluer dans le temps.

Les jeunes vont d'abord "tester" en quelque sorte les squatters, qu'ils perçoivent comme nantis et dont ils comprennent mal la présence dans un squat. C'est le temps des provocations verbales et physiques, de la défiance. Le squat est aussi un espace de vol, ils y dérobent des appareils photos, un ordinateur, et divers objets. Peu à peu pourtant le dialogue entre les deux groupes s'instaure, et les jeunes semblent réaliser que certains squatters ont un passé qui ressemble davantage au leur qu'ils ne le supposaient. Un appareil photos est rendu, des outils restitués. Les jeunes commencent aussi à instrumentaliser l'espace, à le percevoir comme un possible lieu de vie. Bientôt, une clé du squat leur est donnée, ils occupent une pièce où ils passent leurs soirées, entre bières, joints et console vidéo, et entreposent argent et affaires volées dans les recoins de la friche.

59 Les relations entre les deux groupes sont donc empreintes de tensions. Pourtant, des formes de coopération ont également vu le jour, et tout en négociant leur présence avec ses occupants d'origine, les jeunes se sont appropriés le squat. C'est ainsi que l'un d'entre eux menaçait un jour de "mettre le feu » (littéralement) au lieu si la mairie venait à en expulser les artistes. La proximité enfin a produit des moments partagés, sous forme de soirées en musique et de discussions animées, entre des mondes socioculturels qui d'habitude ne se rencontrent pas ${ }^{37}$.

Ces jeunes "étrangers de l'intérieur $»^{38}$ ont parallèlement amené au squat d'autres acteurs de la ville plus disqualifiés encore qu'ils ne le sont, à savoir des mineurs clandestins isolés, et autres migrants primo-arrivants sans logement. Progressivement, le SLAAF est alors devenu un refuge pour des personnes en difficulté qui y passent une nuit, quelques semaines ou s'y installent: une femme seule avec ses enfants, une famille algérienne, des hommes célibataires sans papier passent ou demeurent. La famille dispose d'un réduit au rez-de-chaussée qu'elle habitera pendant plus d'un an, tandis que les hommes partagent à cinq ou six les petites pièces en sous-sol ${ }^{39}$.

61 L'ouverture du SLAAF vers l'extérieur est patente ${ }^{40}$, parfois voulue et planifiée, parfois subie et redoutée, mais toujours tolérée : les frontières du squat vont bien au-delà du Panier. Le squat accueillera successivement ou aux mêmes moments des artistes français et étrangers, des routards, des personnes sans domicile, des jeunes femmes psychotiques, des personnes handicapées. S'y côtoient pendant un temps des architectes canadiens en résidence à Marseille, et des mineurs clandestins marocains en partance pour l'Italie; quelques semaines plus tard une troupe de théâtre vient y répéter le jour, pendant que des SDF y dorment la nuit.

62 Les fêtes organisées par le SLAAF sont à l'image de la population qui l'habite et le fréquente: on y croise des femmes africaines en tenue traditionnelle, des jeunes 
artistes aux styles vestimentaires extravagants, des adolescents du quartier, des couples d'enseignants accompagnés de jeunes enfants, des musiciens berbères ou russes, des hommes algériens qui espèrent trouver dans ces occasions inespérées de rencontre des opportunités de briser un célibat souvent long et subi.

Après plusieurs mois de cohabitation, la lassitude commence à s'emparer des squatters originels, qui constatent que les pourparlers avec la mairie n'aboutissent pas, et aspirent à retrouver calme et stabilité. Peu à peu ils quittent le squat et intègrent d'autres logements, ancien appartement ou chambre chez des amis. Ceux qui restent dans le quartier passent de temps en temps, récupèrent des installations, donnent un coup de main ; puis d'autres projets et activités prennent forme, et l'aventure du SLAAF prend fin. Quelques mois plus tard, ses protagonistes les plus impliqués ouvriront à nouveau un squat, mais à destination de deux familles de demandeurs d'asile cette fois.

Voyages alternatifs

64 Un dernier exemple de squat sur lequel nous nous appuierons pour attester de la diversité de ses acteurs et de leurs capacités d'invention est l'Huilerie occupée, évoquée plus haut avec la famille Ramdani.

L'Huilerie, ainsi nommée par les squatters car le bâtiment abritait auparavant un entrepôt d'huile d'olive, est située dans le quartier Longchamp, quartier du centre-ville anciennement bourgeois et qui conserve un certain prestige malgré sa progressive paupérisation. Elle est habitée pendant toute la durée de son existence ${ }^{41}$ par une majorité de jeunes gens d'origine française et européenne, issus des classes moyennes et populaires.

Ces squatters disent investir des lieux vacants afin de se loger, mais aussi et avant tout pour y vivre des expériences de vie collective autogérées, basées sur le refus du fonctionnement capitaliste et individualiste de la société actuelle. La nourriture est mise en commun, une " caisse commune » sert à assumer les frais de la collectivité, et de nombreuses activités militantes sont organisées (concerts, manifestations de rue, débats etc.). L'Huilerie est divisée en parties communes (dortoir, cuisine, fanzinothèque ${ }^{42}$, bureau, pièce des enfants, garage, salle polyvalente servant tour à tour de lieu de réunion et de fêtes) et privatives (chambres individuelles). Les pièces sont aménagées, repeintes, réhabilitées, sécurisées et l'eau et l'électricité fonctionnent. Elles sont aussi en perpétuelle mutation, régulièrement redécorées, ré-agencées, redéfinies.

De plus, contrairement à la plupart des squatters pour qui le squat ne constitue pas une forme adéquate de présentation de soi ou d'identification, les occupants de l'Huilerie se revendiquent "squatters ", signifiant par là qu'ils l'ont choisi. Le lieu est alors fortement investi, matériellement mais aussi symboliquement. Il est une référence lors de rencontre avec d'autres occupants des squats d'activités. Le squat de l'Huilerie est assimilable à ces espaces que le citadin rend familiers, et dans lesquels il est constitué comme « personne» (Agier, 1999) ${ }^{43}$.

Mais au-delà de ces caractéristiques particulières d'implication et d'identification, nous voudrions insister sur ce que les habitants de l'Huilerie nous apprennent à propos des mobilités transnationales, dans leur forme la plus contemporaine ${ }^{44}$.

Les squatters de l'Huilerie voyagent en effet beaucoup dans les villes d'Europe, à l'occasion de festivals, de manifestations, de rencontres ; ils se rendent également dans des lieux occupés à la campagne, où ils échangent du matériel et des idées. Les contacts avec les squats européens urbains ou ruraux se font notamment grâce à Internet. Des 
listes de diffusion ont été mises en place permettant de faire circuler immédiatement et au plus grand nombre des informations de tout type, appels au soutien de squats menacés d'expulsion, invitations à des rencontres inter-squats etc. La mise en réseau des lieux et des individus est extrêmement prégnante au sein de ces modes d'habiter, emprunts de mouvements et de changements.

L'Huilerie reçoit aussi des jeunes gens venus de toute l'Europe, et d'ailleurs. On y parle quotidiennement français, anglais, allemand, espagnol et italien ${ }^{45}$. Beaucoup de ceux qui arrivent ne savent pas combien de temps ils vont rester. Ils ont appris l'existence du squat dans des conditions très diverses, le plus souvent par ouïe dire, en ayant rencontré ailleurs des squatters de l'Huilerie, ou croisé à Marseille des personnes susceptibles de leur indiquer le lieu. Nombre d'entre eux ne voyagent qu'ainsi : de squat en squat, à travers l'Europe et le monde, sans frais d'hébergement, en entrant en relation avec des groupes de personnes connues ou inconnues, mais avec lesquelles ils se savent en affinité.

71 Ce voyage alternatif se fait par différents moyens, air, terre, mer. Il semble cependant que le mouvement le plus significatif aujourd'hui soit l'acquisition par un nombre toujours croissant d'individus d'un camion, aménagé pour l'habitation, appelé ainsi quelles que soient sa forme et sa taille.

Squat et camion apparaissent comme complémentaires : le squat est le point de chute, le camion le moyen de s'y rendre. Des squats ont été spécialement ouverts en France afin de devenir des espaces d'accueil de "camionneurs ", sortes d'aires de stationnement informelles, qui de plus remplissent une fonction essentielle d'apprentissage de la mécanique, et donc du savoir voyager. C'est ce que nous explique Agathe, occupante de l'Huilerie, au cours d'un entretien :

«Après il y avait un lieu qui s'était ouvert à Bourges, l'Ampérage, parce que Bourges c'est le centre de la France et c'est sur la ligne entre les squatters toulousains, ceux de la Valette, les Parisiens, ceux qui habitent à Lille, ceux qui vont en Espagne, c'était un pur point stratégique. Et puis il y avait une masse de gens qui étaient un peu originaires de ce coin-là, donc il y a un lieu qui s'est ouvert, surtout axé sur les camions, sur la mécanique, justement quand tu tombes en panne, de toutes les façons t'es pas très loin de Bourges, tu vas aller réparer ton camion làbas, et moi j'ai été là-bas pour ça. »

Le camion d'Agathe est ce qui lui permet de voyager, de se sentir en sécurité, et de ne pas dépendre d'un lieu ou des autres. Elle explique qu'elle ne dort pas dans les squats où elle passe, elle y vit, parfois pendant des mois, mais considère qu'elle n'y habite pas : son camion est sa maison ${ }^{46}$.

La stabilité n'est pas forcément là où elle paraît devoir s'imposer, même difficilement : le squat est peut-être donc au final plus fragile qu'un moyen de transport aménagé en foyer, dont on ne risque pas a priori d'être départi demain. La possession d'un moyen de transport habitable a ainsi pour immense avantage d'économiser un investissement matériel et affectif au sein d'un squat dont on sait l'espérance de vie des plus réduites. La mobilité, et la possession d'un véhicule qui en procède peuvent donc paradoxalement être les supports d'une forme de permanence, face à un environnement, squat compris, marqué par l'instabilité.

Les mouvements récents de travellers, ces jeunes gens qui voyagent en Europe dans des camions de ce type et qui ont souvent des pratiques liées à la musique techno et aux raves parties, vont bien au-delà d'une marginalité errante consommatrice de musique 
et de drogues. Certes, on ne peut ignorer qu'évoluent dans ces groupes des jeunes largement désaffiliés, qui sont davantage dans une logique de déshérence que dans celle du voyage. On sait aussi la nécessité d'imaginer des formes souples de logement légal dans lesquelles les plus fragiles d'entre eux pourraient s'arrêter, et bénéficier d'une prise en charge sociale et/ou psychologique. Mais comme les squatters dont il était précédemment question, et avec lesquels ils partagent souvent des espaces et des expériences de vie, ces jeunes associent la possibilité d'être mobiles à la liberté, et revendiquent le droit à l'instabilité spatiale.

Pour Agathe, qui a 30 ans au moment de l'entretien, et qui bénéficie de quelques ressources relationnelles et professionnelles, une sédentarisation trop forte au sein d'un squat peut même entraver cette liberté.

«Et puis après j'ai pas mal tracé... et puis après je me suis dit, je veux plus être tributaire d'un squat, parce qu'à un moment je me suis dit, il y en a marre, je vais vivre dans mon camion, avoir toutes mes affaires dans mon camion, plus avoir besoin de laisser tout mon matos dans un squat, pour pouvoir vraiment être complètement libre à ce niveau-là. Et je me suis dit, donc il me faut un gros camion. Pour mettre tout mon bazar, et quitte à vivre dans un camion, il vaut mieux avoir son petit confort. Il faut mettre son poêle, il faut mettre son labo, il faut prendre sa douche, il faut recevoir ses amis (rires), et il faut pouvoir aller dans les champs, ramasser des champignons, donc je me suis trouvée un camion qui me convenait bien, je me suis achetée un gros camion pour faire tout ce que je voulais faire dedans, et pour pouvoir aller en haut des montagnes avec, aller dans les champs avec, aller partout avec... $»^{47}$.

77 Au final, cette mobilité annexe à son tour des espaces dans la ville ou dans le pays organisés en fonction des besoins de ceux qui vivent sur les routes. On retrouve l'alternance entre mobilité et sédentarité constitutive des mondes des squats; mais le domicile, l'attache territoriale n'est pas là où on l'attendait: le squat a aussi pour vocation de permettre à ceux qui y passent de mieux en repartir.

Ainsi les squats ne sont-ils pas uniquement des lieux d'habitat, mais aussi des points d'ancrage dans des trajectoires transnationales, pour de jeunes Européens qui voyagent selon leurs envies, rencontres et opportunités.

Les squats, espaces de résistance et de mobilité

La majorité des squatters sont des individus qui connaissent des difficultés d'ordre socio-économique et discriminatoire, la non-accession au logement de droit commun étant à la fois cause et conséquence de trajectoires marquées par le déclassement et le déracinement, la perte ou l'affaiblissement des liens familiaux et amicaux. L'ensemble de ces paramètres interagissent et contribuent à la formation de pratiques illégales d'hébergement, qui, parallèlement au travail au noir, permettent aux individus d'éviter le complet dénuement.

Il n'existe cependant pas un monde des squats unifié et uniforme, mais des types de squats habités par des individus aux trajectoires et aux conditions de vie hétérogènes. Les mobilités décrites dans cet article ne sont pas non plus de même nature: leur durée, leur forme, leurs enjeux mêmes ne sont pas comparables, puisqu'il s'agit en simplifiant de trouver un pays où vivre en paix et gagner sa vie pour les premiers, et de vivre des expériences collectives fondées sur la rencontre et la découverte pour les seconds. La situation dans le pays d'origine, les causes du départ, les conditions du voyage, la facilité du retour diffèrent. L'intérêt alors de la mise en parallèle est double : souligner que des mondes sociaux très disparates se croisent, cohabitent et échangent 
au sein d'espaces non planifiés; et rappeler que le squat est toujours indexé à la mobilité, quelle que soit la forme qu'elle prenne, car les migrants savent aussi se saisir de la «mondialisation » et aménager dans et hors les villes qu'ils traversent des espaces et des temps informels de protection, des formes de continuité dans la précarité.

81 Au-delà de la pauvreté, le squat est donc aussi, sous différentes formes, un lieu de résistance: résistance explicite lorsqu'il se donne un contenu politique, dénonce la vacance et revendique des espaces d'autonomie et de création; résistance au dénuement, puisqu'il protège de l'exposition totale de la rue; résistance à la ségrégation, lorsque les migrants précaires occupent un centre urbain que l'on destine désormais aux classes supérieures ; résistance à la discrimination, lorsqu'ils cohabitent avec l'autre; résistance à la dépendance et à l'exploitation qu'incarnent souvent les autres types de logements des précaires; résistance enfin devant l'injonction séculaire faite aux pauvres de se sédentariser. Le squat permet la mobilité de ceux auxquels on interdit de migrer, comme à ceux qui ne peuvent ou ne veulent pas voyager dans les conditions du tourisme ou de l'errance instituées.

\section{BIBLIOGRAPHIE}

AGIER Michel (1999) L'invention de la ville. Banlieues, townships, invasions et favelas, Paris, Éditions des archives contemporaines, $173 \mathrm{p}$.

AUTREMENT (1994) Résister. Le prix du refus, série Morales, 15, mars, 217 p.

ASCARIDE Gilles et CONDRO Salvatore (2001) La ville précaire. Les « isolés » du centre-ville de Marseille, Paris, L'Harmattan, collection Logiques Sociales, 288 p.

BEHAR Daniel (2001) L'intégration à la française, entre rigueur et pragmatisme : le cas des politiques de l'habitat, Hommes et Migrations, 1229, janv-fév., pp. 77-85.

BERNARDOT Marc (1996) La vie des résidents isolés de la Sonacotra, Hommes et Migrations, 1202, oct., pp. 14-22.

BERTONCELLO Brigitte et BREDELOUP Sylvie Éds. (2000) Marseille, carrefour d'Afrique, Hommes et Migrations, 1224, mars-avril, $140 \mathrm{p}$.

BOUILLON Florence (2001a) L'après-guerre de Bosnie : conditions de vie et processus de fragilisation de familles bosno-tsiganes dans une cité marseillaise, Etudes tsiganes,14, pp. 57-70.

BOUILLON Florence (2001b) Des acteurs et des lieux : les économies de la rue à Marseille, in Michel Peraldi Éds., Cabas et containers. Activités marchandes informelles et réseaux migrants transfrontaliers, Paris, Maisonneuve et Larose, pp. 237-268.

BOUILLON Florence (2002) A quoi servent les squats? Compétences des acteurs et ressources des lieux, Revue Française des Affaires Sociales, 2, pp. 45-63.

CHAUVIERE Michel et DURIEZ Bruno Éds. (1992) La bataille des squatters et l'invention du droit au logement. 1945-1955, Paris, Les cahiers du GRMF, 7, 332 p. 
ENGBERSEN Godfried (1999) Sans-papiers. Les stratégies de séjour des immigrés clandestins, Actes de la recherche en sciences sociales, 129, sept., pp. 26-38.

GRAFMEYER Yves (1994) Sociologie urbaine, Paris, Nathan, collection 128, 128 p.

GRAFMEYER Yves (1996) La ségrégation spatiale in Serge Paugam Éd., L'exclusion, l'état des savoirs, Paris, La Découverte, pp. 209-217.

GREEN Nancy L. (2002) Repenser les migrations, Paris, PUF, collection Le nœud gordien, 138 p.

MAZZELLA Sylvie (1996) Le quartier Belsunce à Marseille. Les immigrés dans les traces de la ville bourgeoise, Les Annales de la Recherche Urbaine, 72, pp. 118-125.

MISSAOUI Lamia (2000) Les fluidités de l'ethnicité ou les compétences de l'étranger de l'intérieur, Lille, Éditions du Septentrion, $350 \mathrm{p}$.

PALIDDA Salvatore (1999) La criminalisation des migrants, Actes de la recherche en sciences sociales, 129 , sept., pp. 9-49.

PAUGAM Serge Éds. (1996) L'exclusion, l'état des savoirs, Paris La Découverte, collection Textes à l'appui, $582 \mathrm{p}$.

PERALDI Michel, TARRIUS Alain Éds. (1995) Marseille et ses étrangers, Revue Européenne des Migrations Internationales, vol. XXI, 11, $220 \mathrm{p}$.

PERALDI Michel Éds. (2001) Cabas et containers. Activités marchandes informelles et réseaux migrants transfrontaliers, Paris, Maisonneuve et Larose, $361 \mathrm{p}$.

SAYAD Abdelmalek (1991) L'immigration ou les paradoxes de l'altérité, Paris, Éditions universitaires De Boeck, 331 p.

SEGAUD Marion, BONVALET Catherine, BRUN Jacques Éds. (1998) Logement et habitat : l'état des savoirs, Paris, La Découverte, collection Textes à l'appui, $411 \mathrm{p}$.

SIMON Pierre (1995) Le logement des immigrés, Population et Sociétés, 303, Institut d'Études Démographiques, juillet, $4 \mathrm{p}$.

TARRIUS Alain (2000) Les nouveaux cosmopolitismes. Mobilités, identités, territoires, Paris, Éditions de l'Aube, collection Mondes en cours, $262 \mathrm{p}$.

TARRIUS Alain (2001) Économies souterraines, le comptoir commercial maghrébin de Marseille, Paris, Éditions de l'Aube, collection Mondes en cours, avec la collaboration de Lamia Missaoui, 220 p.

TARRIUS Alain (2002) La mondialisation par le bas. Les nouveaux nomades de l'économie souterraine, Paris, Balland, $169 \mathrm{p}$.

TÉMIME Émile, SAYAD Abdelmalek, JORDI Jean-Jacques (1989-1991) Migrance. Histoire des migrations à Marseille, Aix-en-Provence, Edisud, 4 tomes.

WHITOL DE WENDEN Catherine (2001) Un essai de typologie des nouvelles mobilités, Hommes et Migrations, 1233, sept-oct., pp. 5-12.

\section{NOTES}

1. On utilisera ici le terme migrant comme terme générique pour désigner à la fois les situations d'immigration définitive et celles de la migration temporaire, en conformité avec la loi ou pas. Les « marges » de la ville citées dans le titre sont elles à comprendre dans un sens social et non spacial? 
2. Notamment avec les foyers de travailleurs immigrés dans les années 1950, puis les cités de transit, et le prélèvement d'une partie du $1 \%$ de la taxe des entreprises en faveur du logement des immigrés en 1975. Sur l'évolution des politiques publiques en la matière, cf. D. Béhar (2001).

3. Dont l'accès demeure lui aussi inégalitaire, puisque $58 \%$ des familles issues de l'immigration candidates au logement social obtiennent satisfaction au bout de six mois, contre 75\% de l'ensemble des demandeurs (P. Simon, rapport au Groupe d'études et de lutte contre les discriminations, 2001. Cf. également P. Simon, 1995).

4. Sur ce thème, cf. F. Bouillon, 2001, 2002.

5. Entendue ici dans son sens le plus large comme action de ne pas céder ou de s'opposer à une situation, une difficulté, un danger. Pour différentes approches de la notion de résistance, cf. la revue Autrement (1994).

6. L'Insee note dans son enquête publiée en janvier 2002 sur la population «fréquentant les services d'hébergement et les distributions de repas chauds » que $29 \%$ des Sans Domicile Fixe sont étrangers en 2001 (ils représentent 6,5\% de la population totale), alors même que l'enquête ne prend en considération que les adultes francophones et exclut les squatters et occupants d'habitats de fortune.

7. Sur la nature et la forme des migrations à Marseille, on lira les travaux de B. Bertoncello et S. Bredeloup (2000), S. Mazzella (1996), A. Tarrius (2001), É. Temime (1989-1991), M. Peraldi (2001).

8. Sur les politiques de logement social et d'aide au logement en France, cf. diverses contributions dans les ouvrages de S. Paugam (Éd.) (1996) et C. Bonvalet, J. Brun, M. Segaud (Éds.) (1998).

9. Selon l'expression de R. Park, reprise par Y. Grafmeyer (1994). Ce dernier note également à propos des bailleurs sociaux : « Des notions comme celles de « familles lourdes » ou de « ménages en difficulté » ne désignent pas seulement des problèmes de solvabilité. Bien souvent, elles engagent en même temps, de manière euphémisée, des perceptions discriminatoires concernant la nationalité, l'origine ou le mode de vie. » (Grafmeyer, 1996 : 215).

10. Jusqu'à récemment, seuls étaient exigés la présence d'un point d'eau, l'évacuation des eaux usées, un W.C « même commun » et la " possibilité » d'un chauffage (une prise électrique suffisait donc). Un décret paru le 31/01/02 au Journal Officiel liste désormais un ensemble de normes minimales d'hygiène, de sécurité et de confort qui, si elles ne sont pas respectées, pourront entraîner une baisse du loyer, décidée par voie de justice. Mais encore faut-il que les locataires le sachent, et osent s'en emparer...

11. Cité par M. Henry, «Les 112 taudis de papa Sanchez » in Libération, 2-3 février 2002, pp. 12-13.

12. Sur ce sujet cf. G. Ascaride et S. Condro (2001). Voir aussi A. Sayad (1991) sur les foyers de travailleurs. En mai 2000, pour la première fois, quelques familles soutenues par des associations et des avocats ont porté plainte contre D. Sanchez. En juin 2003 celui-ci a été condamné à 100000 francs d'amende et 2 ans de prison avec sursis. 13. Société Nationale de Construction de Logement pour les Travailleurs, société anonyme d'économie mixte créée en 1956 pour la construction et la gestion de foyers de travailleurs migrants et la résorption de bidonvilles. Sur l'histoire de la Sonacotra, cf. M. Bernardot, 1996.

14. Centre d'Hébergement et de Réinsertion Sociale. Comme son nom l'indique, il a, outre sa fonction d'hébergement, une mission éducative consistant à " réinsérer » l'individu économiquement, socialement et culturellement. 
15. Bénéficiaires du RMI, Revenu Minimum d'Insertion. Celui-ci s'élève à 411,70 euros par mois (au maximum) pour une personne seule au $1^{\mathrm{er}}$ janvier 2003.

16. Aucune condition de revenus n'est exigée à l'entrée d'un CHRS, mais une participation financière est généralement demandée une fois que les droits sociaux de l'individu ont été rétablis.

17. Les locataires en impayés de loyer sont également en illégalité, mais leur situation est très différente car la signature d'un bail est éminemment protectrice en matière de délais avant expulsion et relogement. De plus la «trêve d'hiver ", qui surseoit aux expulsions du $1^{\mathrm{er}}$ novembre au 15 mars ne s'applique pas automatiquement aux squatters, puisque selon l'article 613-3 du code de la construction et de l'habitation, ces dispositions ne sont pas applicables « lorsque les personnes sont entrées dans les lieux par voie de fait ». On peut citer l'exemple de onze familles qui occupaient des appartements de la cité Plan d'Aou à Marseille et en furent expulsées fin février 1999. 18. Selon le dernier recensement Insee, il y a en France, en 1999, 1989758 logements vacants, soit $6,9 \%$ du parc total. À Marseille, on compte 32265 logements vacants, soit $8,3 \%$ du parc, ce qui est inférieur à la majorité des grandes villes françaises (entre 9,5\% et $13 \%)$.

19. Les politiques d'ouverture des frontières aux capitaux et marchandises s'accompagnent de leur fermeture aux hommes issus des pays pauvres, puisque schématiquement, les délocalisations entrepreneuriales exigent aujourd'hui la conservation d'une main-d'œuvre bon marché au sein des pays d'origine et non plus l'importation de cette main-d'œuvre, telle qu'elle fut encouragée jusqu'au milieu des années 1970.

20. Notons que le nombre des demandeurs d'asile a considérablement augmenté ces dernières années, à Marseille comme en France. Aucun dispositif n'est prévu pour les demandeurs d'asile territorial concernant le logement ; ils perçoivent dans le meilleur des cas une aide des Conseils Généraux s'ils ont des enfants en bas âge, pendant quelques mois. L'asile conventionnel donne lui théoriquement droit à un hébergement dans les Centres d'Accueil pour Demandeurs d'Asile (CADA), mais dans les faits les places manquent et une petite minorité y a effectivement accès. Une « allocation d'insertion » est attribuée aux demandeurs non hébergés, équivalente à 270 euros par adulte et par mois, pendant un an.

21. Direction Départementale des Affaires Sanitaires et Sociales.

22. Les habitants du lieu font explicitement la distinction entre ceux qui squattent par choix, auxquels ils s'identifient, et ceux qui squattent par nécessité, et n'ont pas de discours politique plus large accompagnant cette pratique.

23. Mais aussi de Roumanie, de Chine (à Paris particulièrement), de Turquie, du Kosovo...

24. Selon le Rapport d'activités 1998 de l'association « Jeunes errants », qui prend en charge les mineurs clandestins isolés, les origines et les « objectifs » des jeunes placés à l'association suite à une décision judiciaire sont les suivants : 4 sont des enfants de la rue au pays d'origine, c'est-à-dire totalement livrés à eux-mêmes sans référent familial ou parental ; 14 sont des enfants dans la rue au pays d'origine, c'est-à-dire ayant un conflit important avec l'un ou l'autre des parents, vivant une situation de crise ; 15 souhaitent émigrer. Ils ont avant tout le souhait de réussir économiquement et d'envoyer de l'argent au pays ; 5 demandent l'asile politique.

25. Terme explicite employé dans certains pays d'Afrique pour désigner les expulsions des habitants des quartiers illégaux (dits spontanés). 
26. Le projet de loi du gouvernement « pour la sécurité intérieure » prévoyait initialement de faire du squat un nouveau délit, passible de six mois de prison et de 3 000 euros d'amende. Cette disposition a finalement été abandonnée sous la pression des associations de défense des mal-logés.

27. L'ordonnance sur requête est une procédure simplifiée et rapide qui permet de saisir le Président du Tribunal de Grande Instance d'une demande visant à ce que le principe juridique du débat contradictoire ne soit pas respecté. Pour cette raison, elle ne concerne qu'un domaine très limité de cas, lorsque la procédure exige que les individus soient maintenus dans l'ignorance, ou parce qu'il est impossible d'obtenir leur identité, ou encore lorsque la prise de décision présente un caractère d'urgence. Sauf exception, les occupations sans droit ni titre ne répondent pas à ces situations, et plusieurs ordonnances sur requête ont été annulées, suite aux démarches d'un avocat défendant des squatters à Marseille.

28. Le squat est juridiquement assimilé à un domicile à partir du moment où les signes de la domiciliation (matelas, matériel de cuisine...) y sont présents.

29. Confédération Syndicale des Familles. Sa responsable marseillaise actuelle fut une militante active du Mouvement Populaire des Familles (MPF, issu des JOC, Jeunesses Ouvrières Chrétiennes), qui pratiquait à Marseille au sortir de la seconde guerre mondiale la réquisition de bâtiments vides pour y loger des familles sans abris. Pour une généalogie de ces différents mouvements, cf. B. Duriez et M. Chauvière (1992). 30. Elle citait en particulier l'OPAC, Office Public d'Aménagement Concerté, qui dépend du Conseil Général et gère un parc d'environ 26000 logements, et HMP, Habitat Marseille Provence, ancien office municipal HLM qui dépend encore aujourd'hui de la municipalité et gère un parc d'environ 15000 logements.

31. Le juge peut théoriquement octroyer jusqu'à trois ans de délai, mais prononce généralement l'expulsion immédiate. Quelques mois sont parfois accordés, pour laisser le temps aux occupants d'entamer des démarches de relogement.

32. À savoir l'absence de motivation de refus, l'absence de numéro d'attribution de dossier, et l'exigence de HMP que les revenus des locataires potentiels équivalent à 50 francs par personne et par jour au minimum, loyer déduit, ce qui est aussi parfaitement illégal.

33. L'Assistance publique arguera de la solvabilité des squatters pour leur réclamer le versement d'un loyer mensuel de 75000 francs (11 450 euros), soit 1600 francs (245 euros) par famille. Celles-ci devront finalement s'acquitter d'un règlement de 600 francs (92 euros) par mois après décision de justice.

34. Ce qui ne signifie pas que parmi les occupants, aucun n'ait de problème d'accès au logement, et dans la plupart des cas, ces squats assurent aussi une fonction d'hébergement. Mais la rhétorique du choix remplace celle de la contrainte, et la thématique de l'art ou de la politique celle du logement.

35. Les squats d'activités n'ont pas en France l'importance et l'audience qu'ils ont dans d'autres villes européennes, Berlin, Genève, Barcelone et les villes italiennes en particulier. Les squats artistiques les plus connus sont à Paris, et le festival « Art et squats » organisé du 10 septembre au 2 octobre 2002 par le Palais de Tokyo a donné une visibilité médiatique inédite à ce mouvement.

36. La mairie a d'abord conclu un accord oral avec les squatters, qu'elle disait tolérer, avant de se rétracter. Deux explications à ce revirement sont avancées par les squatters : des échéances électorales dépassées, et des tentatives de " récupération » avortées. Le squat est donc appelé à être expulsé. 
37. François, vidéaste du SLAAF, relate ces rapprochements au cours d'un entretien: "On a passé des soirées assez rigolotes avec ceux qui venaient dans la pièce là-bas, mais ils faisaient vraiment leurs trucs... Et il n'y a que certains soirs, en allant les voir, ça a fini par se mêler un peu, ils sont venus dans les chambres, on a joué des percussions et tout ça. Et il y avait des soirs où dans le Grand Atelier il y avait de grosses discussions, les grands frères rentraient, et donc ça discutait vachement. Sur le coup c'était assez compliqué à gérer, mais en fait ça parlait... C'était tout un tas de trucs qu'on défrichait quoi, avec eux, par rapport à eux... Alors ils voyaient un peu ce qu'on faisait... Parce que c'est vrai qu'ils étaient super curieux, ils sont toujours comme ça d'ailleurs, et en même temps vachement farouches quoi (...) Cette histoire qui s'est faite en commun a porté ses fruits, j'en suis sûr, car il y a une histoire du SLAAF dans laquelle ils sont aussi. »

38. Selon l'expression employée par L. Missaoui (2000), qui désigne les Français issus de l'immigration perçus par les autres dans la plus grande altérité.

39. Le lieu fait plusieurs centaines de mètres carrés, mais les deux grands ateliers ne confèrent aucune intimité, et les occupants dorment dans la promiscuité.

40. Si couramment les squats de ce type se proclament « ouverts » aux personnes extérieures, dans les faits l'entrée ou le séjour ne sont généralement pas autorisés à tous, et notamment pas à ceux qui paraissent susceptibles de mettre le groupe en danger : policiers présumés, toxicomanes avérés, individus par trop violents ou psychologiquement perturbés.

41. De février 2000 à janvier 2002. Les squatters ont depuis ouvert d'autres squats dans Marseille, en fonction des affinités qui s'étaient formées à l'Huilerie.

42. Bibliothèque de fanzines, ici anarchistes et libertaires.

43. «Les lieux proches du citadin sont ceux avec lesquels ils s'identifient le plus spontanément, ce sont des espaces de chevauchement presque parfait entre un cadre physique et un sentiment d'appartenance à une collectivité, aussi minime soit-elle, de laquelle il tire sa première forme d'identité face aux autres plus éloignées. » (Agier, 1999 : 33).

44. Pour un approfondissement des formes actuelles des mobilités et des migrations transfrontalières, on lira A. Tarrius (2000, 2002), Nancy L. Green (2002) et C. Whitol de Wenden (2001).

45. Liste des voyages entrepris par des individus ou des groupes habitants de l'Huilerie sur ces deux derniers mois : Grenoble, Toulouse, Paris, Nice, Millau, Montpellier, Barcelone, Gênes, Turin. Liste des villes et pays d'origine des visiteurs du squat sur la même période : Nantes, Lyon, Paris, Lille, Belgique, Allemagne, Angleterre, Pologne, Espagne, Italie, Canada, Maroc, Algérie, Burkina-Faso, Jamaïque, États-Unis. (Notes du journal de terrain, 16 juin 2001).

46. "C'est un peu un cocon un camion et en plus c'est sur coussin d'air, il y a les roues qui t'isolent du sol et qui absorbent les mouvements, les chocs, c'est une bulle, un cocon, et c'est vrai, sortir du cocon quand on est bien... (rires). J'ai pas envie moi. J'emmène partout ma bulle, je suis vachement casanière en fait dans mon camion. À part aller dormir à la belle étoile l'été ou bien je ne sais pas quoi... moi je préfêre dormir là... » (Agathe, Huilerie occupée).

47. Agathe, qui vit donc pourtant dans un camion, ne s'identifie pas aux mouvements travellers évoqués ci-dessus : les identités sont en réalité toujours plus complexes et composites que ne le laissent entendre les sigles univoques de ce type. 


\section{RÉSUMÉS}

Les migrants exclus du logement de droit commun sont aujourd'hui susceptibles de connaître trois types de situation en matière d'hébergement: l'exploitation, la précarité et l'illégalité. Après avoir précisé ce que recouvrent ces termes, cet article s'attache à décrire plus précisément l'expérience de l'illégalité, c'est-à-dire du squat, entendu comme occupation sans droit ni titre d'un logement vacant. La très grande fragilité sociale qu'il indique et qu'il engendre à la fois font du squat un analyseur opérant des mécanismes de production de la misère. Mais son caractère éminemment autonome à l'égard des structures institutionnelles est également révélateur de compétences et de ressources insoupçonnées, tant de la part des migrants en provenance de pays pauvres que de jeunes occidentaux communément désignés comme «marginaux». Structurellement lié à la mobilité, le squat peut au final être lu comme un lieu de résistance à la pauvreté, ainsi qu'à l'injonction de sédentarisation faite aux pauvres de nos sociétés.

Migrants and Squats: destitution and resistance in the city's fringes. Migrants excluded from the legal housing system in France are particularly susceptible to exploitation, destitution and illegality. After clarifying the meaning of these terms, this article seeks to detail the experience of illegality - that is " the squat ", understood as occupation without right or title to a vacant flat. Squatting is both an effect of poverty and a contributing cause of poverty. Analyzing the phenomenon of the squat helps explain the mechanisms of urban misery. But it also explains unexpected skills and resourcefulness of migrant populations from the developing world, as well as of "disaffected» youth, that exist beyond public institutions and official policies. Structurally linked to space mobility, the squat can be viewed as a place of resistance by the impoverished to their destitution as well as to society's efforts to keep them settled in one place.

Emigrantes y ocupación : precariedades y resistencias en las márgenes de la ciudad. En lo que al alojamiento se refiere, los inmigrantes excluidos del derecho común a la vivienda se enfrentan, hoy en día, a tres tipos de situaciones: la explotación, la precariedad y la ilegalidad. Tras haber precisado el significado de dichos términos, este artículo se centra, más concretamente, en la descripción de la experiencia de la ilegalidad; del « squat », entendido como la ocupación de una vivienda vacante sin derecho ni título. La gran fragilidad social que indica y que engendra el "squat", lo convierten en un mecanismo de producción de miseria. Mas, al mismo tiempo, su eminente autonomía respecto de las estructuras institucionales es reveladora de las competencias y de los recursos insospechados con los que cuentan los inmigrantes originarios de países pobres o los jóvenes occidentales comúnmente calificados de «marginales ». Estructuralmente ligado a la movilidad, el « squat » puede ser identificado como un lugar de resistencia a la pobreza y a la sedentarización de los pobres de nuestra sociedad.

\section{AUTEUR}

\section{FLORENCE BOUILLON}

ATER en sociologie à l'Université de la Méditerranée (Aix-Marseille II), Doctorante au SHADYC - École des Hautes Études en Sciences Sociales, Centre de la Vieille Charité, 2 rue de la Charité, 13002 Marseille. 